\title{
Erratum a: Analisi di costo efficacia dei nuovi anticoagulanti orali nella prevenzione dell'ictus in pazienti con fibrillazione atriale non valvolare in Italia
}

\author{
Erratum to: Cost efficacy analysis of new oral anticoagulant for stroke prevention \\ in non-valvular atrial fibrillation in Italy
}

\section{Roberto Ravasio • M.P. Pedone • M. Ratti}

Pubblicato online: 10 dicembre 2014

(C) Springer International Publishing Switzerland 2014

Erratum a: PharmacoEcon Ital Res Artic (2014) 16:22 DOI 10.1007/s40276-014-0022-x

La versione originale di questo articolo contiene alcune inesattezze, di seguito specificate.

(1) Pagina 2 di 10, seconda colonna, riga 11; pagina 3 di 10, Figura 1 e didascalia; pagina 3 di 10, seconda colonna, riga 6: in luogo dell'acronimo "MIA" si legga "AMI".

(2) Pagina 2 di 10, seconda colonna, riga 13 dal basso; pagina 5 di 10, prima colonna, riga 1 dal basso; pagina 5 di 10, se conda colonna, riga 2 dal basso: in luogo dell'acronimo "IMA" si legga "AMI".

(3) Pagina 5 di 10, Tabella 2: la dicitura "Rischio relativo (vs. Warfarin)" si riferisce solo alle tre colonne "Dabigatran", "Rivaroxaban" e "Apixaban"; sopra la colonna "Warfarin" va posta la dicitura "Rischio annuo". Inoltre il trattino sostituisce la virgola tra i valori all'interno delle parentesi quadre. Di seguito si riporta la Tabella corretta.
(4) Pagina 6 di 10, Tabella 4:

Sezione "Evento clinico", riga 8: anziché "Ictus ischemico, disabilità "indipendente",, si legga "Emorragia intracranica, disabilità "indipendente",

Sezione "Evento clinico", riga 9: anziché "Ictus ischemico, disabilità "moderata",", si legga "Emorragia intracranica, disabilità "moderata",

Sezione "Evento clinico", riga 10: anziché "Ictus ischemico, disabilità "dipendente",", si legga "Emorragia intracranica, disabilità "dipendente","

Di seguito si riporta la Tabella corretta.

(5) Pagina 7 di 10, prima colonna, riga 7: anziché "versione 2103", si legga "versione 2013".

(6) Pagina 9 di 10, seconda colonna, righe 15-16: anziché "in termini di dosaggi, monitoraggio, comorbidità, ecc." si legga "in termini di compliance, comorbidità, ecc.".

Si porgono scuse ai Lettori per le inesattezze occorse.

The online version of the original article can be found under doi:10.1007/s40276-014-0022-x.

\footnotetext{
R. Ravasio ( $\varangle)$

Springer Healthcare Italia srl, Via Lanino, 5, 20144, Milano, Italia e-mail: roberto.ravasio@pharmes.it
}

M.P. Pedone · M. Ratti

Boehringer Ingelheim Italia s.p.a, Milano, Italia 
Tabella 2 Rischi relativi associati agli eventi clinici

\begin{tabular}{|c|c|c|c|c|}
\hline \multirow[t]{2}{*}{ Parametri } & \multirow{2}{*}{$\frac{\text { Rischio annuo }}{\text { Warfarin }}$} & \multicolumn{3}{|c|}{ Rischio relativo (vs. Warfarin) } \\
\hline & & Dabigatran & Rivaroxaban & Apixaban \\
\hline \multicolumn{5}{|l|}{ IS } \\
\hline $\mathrm{CHADS}_{2}=\mathbf{0}$ & $0,62 \%$ & & & \\
\hline 1 & $0,77 \%$ & & & \\
\hline 2 & $1,01 \%$ & $0,71[0,53-0,96]$ & $0,95[0,76-1,18]$ & $0,80[0,65-1,00]$ \\
\hline $3-4$ & $1,75 \%$ & & & \\
\hline $5-6$ & $3,34 \%$ & & & \\
\hline SE & $0,18 \%$ & $0,55[0,25-1,24]$ & $0,26[0,10-0,71]$ & $0,76[0,62-0,86]$ \\
\hline TIA & $0,84 \%$ & $0,79[0,59-1,07]$ & Assunto uguale a dabigatran & \\
\hline ICH (inclusi HS) & $0,80 \%$ & $0,28[0,17-0,45]$ & $0,72[0,51-1,01]$ & $0,42[0,30-0,58]$ \\
\hline ECH & $2,84 \%$ & $1,07[0,91-1,26]$ & $1,21[1,04-1,41]$ & $0,79[0,68-0,93]$ \\
\hline Sanguinamenti minori & $16,37 \%$ & $0,87[0,82-0,93]$ & Assunto uguale a dabigatran & \\
\hline AMI & $0,64 \%$ & $1,28[0,95-1,74]$ & $0,94[0,73-1,22]$ & $0,88[0,66-1,17]$ \\
\hline $\begin{array}{l}\text { Rischio relativo di } \\
\text { futuri eventi AMI }\end{array}$ & 3,11 & & & \\
\hline
\end{tabular}

Tabella 4 Valorizzazione della terapia farmacologica, degli eventi clinici e del follow-up

\begin{tabular}{|c|c|c|}
\hline Descrizione prestazione & Costo $(€)$ & Fonte \\
\hline \multicolumn{3}{|l|}{ Terapia farmacologica } \\
\hline Aspirina (dose die 100 mg) & 0,04 & {$[28]$} \\
\hline Dabigatran etexilato (dose die $300 \mathrm{mg}$ ) & 2,10 & {$[28]$} \\
\hline Dabigatran etexilato (dose die $220 \mathrm{mg}$ ) & 2,10 & {$[28]$} \\
\hline Rivaroxaban (dose die 20 mg) & 2,07 & {$[28]$} \\
\hline Apixaban (dose die 10 mg) & 2,10 & {$[28]$} \\
\hline Warfarin (dose die $5 \mathrm{mg}$ ) & 0,04 & {$[28]$} \\
\hline \multicolumn{3}{|l|}{ Evento clinico } \\
\hline Ictus ischemico fatale & $4.000,00$ & Lucioni et al. [30] \\
\hline Ictus ischemico, disabilità "indipendente" & $9.350,00$ & Lucioni et al. [30] \\
\hline Ictus ischemico, disabilità "moderata" & $14.500,00$ & Lucioni et al. [30] \\
\hline Ictus ischemico, disabilità "dipendente" & $27.500,00$ & Lucioni et al. [30] \\
\hline Embolia sistemica & $2.549,50$ & DRG78; DRG131 \\
\hline Attacco ischemico transitorio & $2.543,00$ & DRG524 \\
\hline Emorragia intracranica o ictus emorragico fatale & $4.000,00$ & Ipotesi stesso costo IS \\
\hline Emorragia intracranica, disabilità "indipendente" & $9.350,00$ & Ipotesi stesso costo IS \\
\hline Emorragia intracranica, disabilità "moderata" & $14.500,00$ & Ipotesi stesso costo IS \\
\hline Emorragia intracranica, disabilità "dipendente" & $27.500,00$ & Ipotesi stesso costo IS \\
\hline Emorragia extra-cranica & $6.940,80$ & Capri et al. [31] \\
\hline Sanguinamento minore & 130,00 & Marchetti et al. [32] \\
\hline Infarto del miocardio acuto, fatale & $4.018,00$ & DRG123 \\
\hline Infarto del miocardio acuto, non fatale & $4.038,50$ & DRG121; DRG122 \\
\hline \multicolumn{3}{|l|}{ Follow-up (costo per trimestre) } \\
\hline Disabilità indipendente con storia di ictus & 245,30 & Lucioni et al. [30] \\
\hline Disabilità moderata & 332,00 & Lucioni et al. [30] \\
\hline Disabilità dipendente & 434,00 & Lucioni et al. [30] \\
\hline Infarto del miocardio & 172,50 & Berto et al. [33] \\
\hline
\end{tabular}

